University of New Orleans

ScholarWorks@UNO

$12-1-1986$

\title{
Response of the rotating-detector ellipsometer to incident partially polarized light
}

R. M.A. Azzam

University of New Orleans, razzam@uno.edu

Follow this and additional works at: https://scholarworks.uno.edu/ee_facpubs

Part of the Electrical and Electronics Commons

\section{Recommended Citation}

R. M. A. Azzam, "Response of the rotating-detector ellipsometer to incident partially polarized light," Appl. Opt. 25, 4224-4225 (1986)

This Article is brought to you for free and open access by the Department of Electrical Engineering at ScholarWorks@UNO. It has been accepted for inclusion in Electrical Engineering Faculty Publications by an authorized administrator of ScholarWorks@UNO. For more information, please contact scholarworks@uno.edu. 


\section{Response of the rotating-detector ellipsometer to incident partially polarized light}

\section{R. M. A. Azzam}

University of New Orleans, Department of Electrical Engineering, Lakefront, New Orleans, Louisiana 70148.

Received 16 June 1986.

0003-6935/86/234224-02\$02.00/0.

(C) 1986 Optical Society of America.

A unique ellipsometer that uses only a photodetector and no other optical element has been described recently. ${ }^{1}$ The detector surface is partially specularly reflecting and intercepts the incoming light beam at oblique incidence (Fig. 1). A fraction of the incident radiation is absorbed which depends on the incident polarization and the azimuthal orientation of the plane of incidence (POI). The detector is synchronously rotated around the light beam as an axis so that the POI becomes a revolving plane. The output electrical signal $i_{d}$ of the linear photodetector is proportional to the amount of absorbed radiation and is modulated by the rotation. It is this modulation that determines the input state of polarization.

The analysis of Ref. 1 was carried out for incident totally polarized light, and it was found that this rotating-detector ellipsometer (RODE) determines the elliptic vibration of the electric vector except for handedness. In this Letter we consider the response of RODE to partially polarized incident light which is described more generally by the four Stokes parameters $S_{0}, S_{1}, S_{2}$, and $S_{3}$. We prove that RODE can determine the first three Stokes parameters $S_{0}, S_{1}$, and $S_{2}$, but that it is totally blind to $S_{3}$ (hence to the degree of circular polarization) as may be intuitively anticipated. Because radiation from the sun-lit atmosphere and many astronomical sources is mostly partially linearly polarized (see, e.g., Refs. 2-4), a vast range of possible applications is open to RODE.

One position of the detector, established, e.g., when the reflected beam passes through a fixed aperture, defines a reference POI. Two axes, $x$ and $y$, parallel and perpendicular to that reference plane, define the coordinate system with respect to which the Stokes parameters of incident light are defined. These parameters are lumped in the unknown Stokes vector

$$
\mathbf{S}_{i}^{x y}=\left[\begin{array}{l}
S_{0} \\
S_{1} \\
S_{2} \\
S_{3}
\end{array}\right]
$$

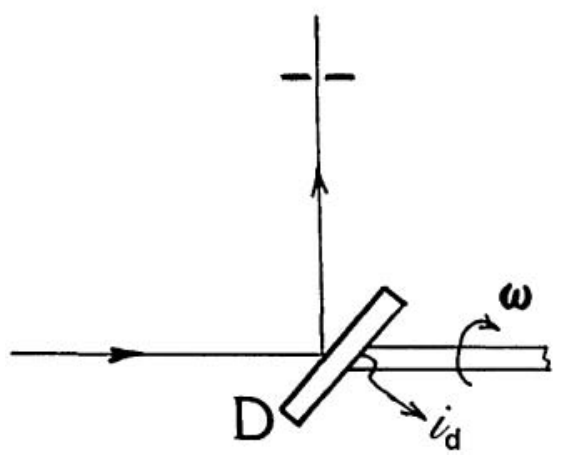

Fig. 1. Rotating-detector ellipsometer, after Ref. 1.
At an arbitrary moment, the directions parallel $p$ and perpendicular $s$ to the instantaneous POI make an angle $\theta$ with the $x$ and $y$ axes, respectively. The Stokes vector of incident light referenced to the rotated $p s$ coordinate system is given by

$$
\mathbf{S}_{i}^{p s}=\mathbf{R}(\theta) \mathbf{S}_{i}^{x y},
$$

where

$$
\mathbf{R}(\theta)=\left[\begin{array}{cccc}
1 & 0 & 0 & 0 \\
0 & \cos 2 \theta & \sin 2 \theta & 0 \\
0 & -\sin 2 \theta & \cos 2 \theta & 0 \\
0 & 0 & 0 & 1
\end{array}\right]
$$

is the Mueller matrix of a coordinate rotation $\theta$. The Stokes vector of the reflected light is given by

$$
\begin{aligned}
\mathbf{S}_{r}^{p s} & =\mathbf{M} \mathbf{S}_{i}^{p s} \\
& =\mathbf{M R}(\theta) \mathbf{S}_{i}^{x y},
\end{aligned}
$$

where $\mathbf{M}$ is the Mueller matrix that represents the effect of reflection from the detector surface and is given by

$$
\mathbf{M}=\bar{R}\left[\begin{array}{cccc}
1 & -\cos 2 \psi & 0 & 0 \\
-\cos 2 \psi & 1 & 0 & 0 \\
0 & 0 & \sin 2 \psi \cos \Delta & \sin 2 \psi \sin \Delta \\
0 & 0 & -\sin 2 \psi \sin \Delta & \sin 2 \psi \cos \Delta
\end{array}\right] .
$$

In Eq. (5), $\bar{R}$ is the intensity reflectance for incident unpolarized or circularly polarized light, and $\tan \psi \exp (j \Delta)$ gives the ratio of the complex amplitude $p$ and $s$ reflection coefficients.

We need only know the first Stokes parameter $S_{0 r}$ of the reflected beam (that represents its flux). Substituting the matrices given by Eqs. (1), (3), and (5) into the right-hand side of Eq. (4) and carrying out the matrix multiplications, we obtain

$$
S_{0 r}=\bar{R}\left[S_{0}-(\cos 2 \psi \cos 2 \theta) S_{1}-(\cos 2 \psi \sin 2 \theta) S_{2}\right] .
$$

The detected signal is given by

$$
i_{d}=k\left(S_{0}-S_{0 r}\right),
$$

where $k$ is the detector sensitivity parameter, and $\left(S_{0}-S_{0 r}\right)$ is the absorbed flux. If Eq. (6) is used in Eq. (7), we get

$$
\begin{aligned}
i_{d}= & k\left[(1-\bar{R}) S_{0}+(\bar{R} \cos 2 \psi) \cos 2 \theta S_{1}\right. \\
& \left.+(\bar{R} \cos 2 \psi) \sin 2 \theta S_{2}\right] .
\end{aligned}
$$

Equation (8) shows that RODE responds to the first three Stokes parameters $S_{0}, S_{1}$, and $S_{2}$ but that it is totally blind to $S_{3}$. With $\theta=\omega t$, where $\omega$ is the angular rotation frequency and $t$ is the time, it is evident that $S_{0}, S_{1}$, and $S_{2}$ appear in the amplitudes of distinct Fourier components of the modulated signal $i_{d}$ and, therefore, can be separately determined. As may be expected, Eq. (8) is very similar to what one gets with the rotating-analyzer ellipsometer. ${ }^{6}$ To extract $S_{0}, S_{1}$, and $S_{2}$, the detector parameters $k, \bar{R}$, and $\psi$ must be known or measured by calibration.

Rarely are we interested in the absolute values of the Stokes parameters. This calls for normalization of Eq. (8) by factoring out the constant ( $\theta$-independent) term; this gives

$$
i_{d}=i_{0}(1+a \cos 2 \theta+b \sin 2 \theta),
$$

where

$$
\begin{aligned}
& i_{0}=k(1-\bar{R}) S_{0}, \\
& a=\alpha s_{1}, \quad b=\alpha s_{2},
\end{aligned}
$$




$$
\begin{aligned}
\alpha & =\bar{R} \cos 2 \psi /(1-\bar{R}), \\
s_{1} & =S_{1} / S_{0}, \quad s_{2}=S_{2} / S_{0} .
\end{aligned}
$$

Digital Fourier analysis of $i_{d}$ determines the cosine and sine amplitudes $a$ and $b$, and from Eqs. (11) one gets the normalized Stokes parameters

$$
s_{1}=a / \alpha, \quad s_{2}=b / \alpha .
$$

The single important parameter $\alpha$ can be determined in a simple calibration step in which RODE is illuminated by totally linearly polarized light of arbitrary azimuth (by placing a good linear polarizer in the path of the incident beam), and the corresponding Fourier amplitudes $a_{c}$ and $b_{c}$ of the output calibration signal are recorded. Then we can use the fact that

$$
s_{1}^{2}+s_{2}^{2}=1
$$

for total incident linear polarization to obtain, using Eqs. (14),

$$
\alpha=\left(a_{c}^{2}+b_{c}^{2}\right)^{1 / 2} .
$$

The calibration signal can be put in the form $i=i_{0}[1+\alpha$ $\cos \left(2 \theta-2 \theta_{0}\right)$ ], where $\theta_{0}$ is the azimuth of the incident linear vibration from the reference plane and $\alpha$ represents the modulation depth. This identifies $\alpha$ with the parameter $m_{L}$ of Ref. 1. This can also be verified by manipulating Eq. (12) as follows. Let $R_{p}$ and $R_{s}$ be the detector surface reflectances for incident $p$ - and $s$-polarized light. Then we can write

$$
\begin{aligned}
\bar{R} & =1 / 2\left(R_{p}+R_{s}\right), \\
\cos 2 \psi & =\left(1-\tan ^{2} \psi\right) /\left(1+\tan ^{2} \psi\right) \\
& =\left[1-\left(R_{p} / R_{s}\right)\right] /\left[1+\left(R_{p} / R_{s}\right)\right] \\
& =\left(R_{s}-R_{p}\right) /\left(R_{s}+R_{p}\right) .
\end{aligned}
$$

If $\bar{R}$ and $\cos 2 \psi$ in Eq. (12) are replaced by their values from Eqs. (17), one gets

$$
\begin{aligned}
\alpha & =\left(R_{s}-R_{p}\right) /\left(2-R_{s}-R_{p}\right) \\
& =m_{L} .
\end{aligned}
$$

It should be noted that $\alpha$ is a function of wavelength ${ }^{1}$ and must be measured over the useful spectral range of the detector for spectroscopic applications.

To summarize: we have shown that the rotating-detector ellipsometer (RODE), first described in Ref. 1, can be used to measure the first three Stokes parameters of incident light which is in a general state of partial polarization. This considerably widens the scope of possible applications of this unique and simple instrument.

The author sincerely appreciates the kind hospitality of the Département de Physique du Solide, Université de Provence, Marseille, France, where this work was done. A Fulbright Senior Research Scholar Award is also gratefully acknowledged.

\section{References}

1. R. M. A. Azzam, "Rotating-Detector Ellipsometer for Measurement of the State of Polarization of Light," Opt. Lett. 10, 427 (1985).

2. T. Geherls, Ed., Planets, Stars and Nebulae Studied with Photopolarimetry (U. Arizona Press, Tucson, 1974).
3. D. L. Coffeen, "Polarization and Scattering Characteristics in the Atmospheres of Earth, Venus, and Jupiter," J. Opt. Soc. Am. 69, 1051 (1979).

4. T. Prosch, D. Hennings, and E. Raschke, "Video Polarimetry: A New Imaging Technique in Atmospheric Science," Appl. Opt. 22, 1360 (1983).

5. R. N. A. Azzam and N. M. Bashara, Ellipsometry and Polarized Ligh: (North-Holland, Amsterdam, 1977), p. 491.

6. P.S. Hauge, "Survey of Methods for the Complete Determination of the State of Polarization," Proc. Soc. Photo-Opt. Instrum. Eng. 88, 3 (1976).

\section{Polarizing beam splitters for infrared and millimeter waves using single-layer-coated dielectric slab or unbacked films}

\section{R. M. A. Azzam}

University of New Orleans, Department of Electrical Engineering, Lakefront, New Orleans, Louisiana 70148. Received 16 June 1986.

0003-6935/86/234225-03\$02.00/0.

(C) 1986 Optical Society of America.

An ideal polarizing beam splitter ${ }^{1,2}$ (PBS) is a device that separates two cotraveling orthogonally linearly polarized components of an incident light beam (usually denoted by $p$ and $s$ ) into two beams that propagate in different directions, with each split beam being purely polarized in one of the original orthogonal linear states. Practical PBSs are based on the phenomenon of double refraction, crystal polarizers such as the Rochon and Wollaston prisms, ${ }^{1}$ or interference in a multilayer coating embedded inside a (glass) cube..$^{1,2}$

The objective of this paper is to describe a simple alternative PBS (Fig. 1) that consists of a plane-parallel optically isotropic dielectric slab of refractive index $n_{2}$ that is as low as possible, which is coated on both sides by a transparent thin film of refractive index $n_{1}$ that is as high as possible. The slab is oriented so that light is incident (from air, $n_{0}=1$ ) at an angle $\phi_{p}=\arcsin \left(u^{1 / 2}\right)$, where $u$ is the root between 0 and 1 of the quadratic equation ${ }^{3}$

$$
\begin{aligned}
a u^{2}+b u+c & =0, \\
a=n_{1}^{8}-n_{2}^{4}, \quad b & =2 n_{1}^{2} n_{2}^{4}-n_{1}^{8}\left(n_{2}^{2}+1\right), \\
c & =n_{1}^{4} n_{2}^{2}\left(n_{1}^{4}-n_{2}^{2}\right) .
\end{aligned}
$$

At $\phi_{p}$ the $p$-polarized component (parallel to the plane of incidence) is totally transmitted $\left(R_{p}=0, T_{p}=1\right)$ when the thickness of the film equals

$$
d_{p}=(\lambda / 4)\left(n_{1}^{2}-u\right)^{-1 / 2}
$$

or an odd multiple thereof. To achieve an acceptable PBS the $s$ component must be nearly totally reflected $\left(R_{s} \approx 1\right.$ and $T_{s} \approx 0$ ). The intensity reflectance for the $s$ polarization of one coated surface takes the simple form

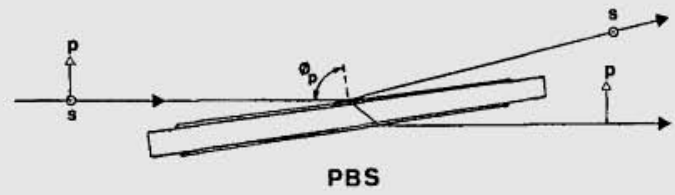

Fig. 1. Polarizing beam splitter using a coated dielectric slab. A very small $s$ component in the transmitted beam is not indicated. 